\title{
Traitements de l'échantillon biologique avant l'analyse chromatographique. Applications à la pharmacocinétique et à la toxicologie
}

\section{Sample handling and pre-treatment prior to chromatographic analysis. Applications to pharmacokinetics and toxicology}

Olivier NICOLAS ${ }^{(1)}$, Damien ROBERT ${ }^{(1)}$, Marie T. KELLY ${ }^{(2)}$, Françoise BRESSOLLE ${ }^{(1) *}$

(1) Laboratoire de Pharmacocinétique Clinique

(2) Centre de Formation en Enologie, Faculté de Pharmacie, Université Montpellier I 34093 Montpellier Cedex - FRANCE

* Auteur à qui adresser la correspondance : Dr Françoise BRESSOLLE, Laboratoire de Pharmacocinétique Clinique, Faculté de Pharmacie - BP 14491, Université Montpellier I - 34093 Montpellier Cedex - FRANCE

Tél : 33467548075 - Fax : 33467548075 - E-mail : FBressolle@aol.com

\section{$R E ́ S U M E$}

Lors de la quantification des médicaments (et de leurs métabolites éventuels) dans les milieux biologiques, deux étapes sont importantes: $i)$ le recueil et la conservation de l'échantillon qui doivent garantir la stabilité des analytes et ii) le prétraitement de celui-ci. Cette dernière étape est particulièrement cruciale lors d'expertises médico-légales. Les difficultés rencontrées sont liées à la complexité du milieu biolo. gique mais aussi aux faibles concentrations du ou des analytes à quantifier. Après un bref rappel des méthodes d'analyse les plus couramment utilisées pour quantifier les médicaments et des problèmes liés au recueil des échantillons biologiques, nous présenterons les différentes méthodes de pré-traitement en vue d'une analyse par chromatographie liquide haute performance ou par chromatographie en phase gazeuse. Les avantages et les inconvénients de ces méthodes et leur possibilité d'automatisation seront discutés.

\section{MOTS-CLÉS}

Matrice biologique, pré-traitement, déprotéinisation, extraction liquide-liquide, extraction solide-liquide.

\section{SUMMARY}

During the analysis of drugs and metabolites in biological matrices, two important steps must be considered, i) the stability of the compounds during sample collection and storage and ii) sample pretreatment. This later is a particularly crucial problem in the case of forensic analysis. The difficulties encountered are related to the complexity of the biological matrix and to the fact that the analyte(s) are frequently present in extremely low concentrations. Following a brief summary of the various methods routinely used in drug analysis and the problems encountered during sample collection and storage, different techniques employed in the preparation of biological samples prior to separation by liquid or gas chromatography will be presented. The advantages and disadvantages of the techniques described will be discussed in addition to the various possibilities for automation.

\section{KEY-WORDS}

Biological matrix, Pre-treatment, Deprotenisation, LiquidLiquid extraction, Solid-phase extraction. 


\section{Introduction}

Au cours des études de pharmacocinétique, de métabolisme et en toxicologie clinique, il est nécessaire de quantifier les médicaments et leurs métabolites éventuels dans des milieux biologiques complexes comme, par exemple, le sang total, le plasma, le sérum, les urines, la bile, les fèces, les tissus, etc $(1,2)$. Ces matrices contiennent une multitude de substances endogènes (protéines dans le plasma ou acides gras dans les urines) en quantités bien supérieures à celles des composés et de leurs métabolites à quantifier. Nombreux sont les composés endogènes présentant des groupements fonctionnels réactifs (comme les fonctions carboxyliques des acides aminés ou des acides gras) pouvant participer aux réactions de dérivation et interférer avec l'analyse chromatographique des composés d'intérêt. Le pré-traitement des échantillons est donc une étape fondamentale pour ce type d'analyse. Il devient particulièrement crucial lorsqu'il s'agit de rechercher des médicaments dans des milieux biologiques issus de cadavre au cours d'expertises médicolégales (3). Il a été rapporté que les concentrations d'acides palmitique, linoléique et stéarique, de diocyl phtalate, de squalène et de cholestérol augmentent au fur et à mesure de la putréfaction (4).

Après un bref rappel des méthodes d'analyse les plus couramment utilisées pour quantifier les médicaments et des problèmes liés au recueil des échantillons (il est important en effet de préciser dans quelles conditions ces derniers doivent être recueillis), nous présenterons les techniques de pré-traitement avec leurs avantages, leurs inconvénients et les possibilités d'automatisation. Afin d'illustrer les différents chapitres de ce manuscrit, des articles publiés dans le domaine de la pharmacocinétique et de la toxicologie seront donnés en exemple.

\section{Méthodes d'analyse}

Les méthodes les plus couramment utilisées sont la chromatographie en phase gazeuse (CG) avec détection par ionisation de flamme, thermoînique, capture d'électron ou spectrométrie de masse (SM) et la chromatographie liquide haute performance (CLHP) avec. détection dans l'ultraviolet (UV), en fuorescence ou par électrochimie. Mais en raison du caractère non volatil de la plupart des médicaments, l'analyse par CG nécessite une dérivatisation préalable du composé. Par contre, la CLHP est particulièrement bien adaptée à l'analyse des substances non volatiles et à leurs métabolites plus polaires.

En CLHP, l'élimination des composants endogènes dus à la matrice est particulièrement importante car ces der- niers provoquent une détérioration rapide des performances de la colonne analytique (baisse du nombre de plateaux théoriques, perte de sélectivité, élargissement des pics) et du détecteur (saturation, augmentation du bruit de fond). En effet, ces composés peuvent s'adsorber irréversiblement sur la phase stationnaire comme les lipides (perte de sélectivité) ou précipiter dans la colonne comme les protéines (augmentation de la pression en tête de colonne). L'utilisation de pré-colonnes placées juste avant la colonne analytique permet de limiter partiellement ces problèmes.

Il faut également souligner que le choix de la méthode d'extraction peut être fonction du mode de détection choisi. C'est ainsi que les extraits obtenus devront être particulièrement propres lorsque l'analyse est réalisée par CLHP-UV à basse longueur d'onde (210-220 nm) ou par CG et détection par capture d'électron.

Depuis quelques années la CLHP couplée à la spectrométrie de masse ou à la spectrométrie de masse en tandem est de plus en plus utilisée. Cependant, une des limites de cette méthode est sa susceptibilité à l'effet matrice $(5,6)$. L'effet matrice va perturber l'analyse qualitative et quantitative et par conséquent risque de compromettre la validité des résultats obtenus $(7,8)$. L'effet matrice va dépendre du mode d'analyse, « full scan » ou «SIM ». Dans le mode « full scan » l'addition d'ions issus de la matrice risque de contaminer le spectre de masse de la molécule cible. Dans le mode « SIM , les interférences peuvent être détectées par des rapports d'ions non valides lorsque les composés interférents présentent des ions en commun avec le composé à doser.

\section{Problèmes liés au recueil des échantillons biolo- giques avant l'étape de pré- traitement}

Avant d'étudier les différentes techniques de pré-traitement, il est important de préciser dans quelles conditions l'échantillon à analyser doit être recueilli. Bien que l'analyste n'ait pas la responsabilité du recueil de l'échantillon, il devra communiquer la procédure à respecter qui garantira son intégrité par rapport à l'analyse qui sera réalisée ultérieurement.

Les conditions de recueil de l'échantillon (sang total, plasma, sérum, urine, bile, etc) sont souvent définies en fonction des propriétés de l'analyte et en particulier sa stabilité au cours du processus de conservation et au cours de l'analyse elle-même. 
Dans l'organisme, le $\mathrm{pH}$ sanguin est maintenu entre 7,35 et 7,45 ; cet équilibre est contrôlé essentiellement par la vitesse de ventilation pulmonaire (11-13). Exvivo, le sang ou le plasma ne possédant pas ce système de régulation, du $\mathrm{CO}_{2}$ est régulièrement perdu pendant les étapes de stockage et de manipulation. Des pertes de $\mathrm{CO}_{2}$ sont également observées lors de la conservation d'échantillons de bile. Le résultat est une augmentation du $\mathrm{pH}$ de la matrice. La liaison aux protéines plasmatiques des médicaments est souvent $\mathrm{pH}$ dépendante. Par conséquent, toute variation de $\mathrm{pH}$ ex-vivo peut modifier de façon significative le pourcentage de liaison d'un composé aux protéines plasmatiques (1113). La stabilité des composés peut également être modifiée par les changements de $\mathrm{pH}$ (14). Par exemple, les composés à fonctions esters, amides, carbamates, urée, béta-lactame, lactones, hydantoines, glucuronides acylés, etc sont susceptibles d'être dégradés (10). Le pH de l'échantillon biologique peut être stabilisé à 7,47,5 par addition de tampon citrate ou phosphate (10). Par conséquent, le maintien d'un pH stable est essentiel lorsqu'il s'agit de caractériser et de quantifier des composés qui sont eux mêmes sensibles au $\mathrm{pH}$ ou qui génèrent des métabolites thermosensibles.

Il est parfois nécessaire de quantifier un composé dans les urines (stupéfiants par exemple). Pour le recueil des urines des conservateurs sont souvent nécessaires.

Les cheveux et les ongles sont surtout utilisés en toxicologie médico-légale pour détecter une exposition à des poisons ou à des « substances » illicites. Le site du prélèvement doit être noté puisque par exemple la vitesse de pousse des cheveux n'est pas la même suivant les zones du cuir chevelu.

Il est parfois nécessaire de doser un composé dans les tissus. Il peut s'agir de biopsies obtenues par chirurgie, dans ce cas la quantité de tissu disponible pour l'analyse est très faible et l'échantillon est toujours contaminé par du sang. Un dosage d'hémoglobine permet de corriger cette contamination. Il est important de noter également le site du prélèvement. Le prélèvement tissulaire peut correspondre à la totalité de l'organe (toxicocinétique, expertise médico-légale), dans ce cas l'organe sera broyé de façon à obtenir un homogénat. A cet effet, des homogénéisateurs type Ultraturax ${ }^{\circledR}$ peuvent être utilisés. Cependant, le facteur limitant est la thermosensibilité des molécules. Afin d'éviter toute dégradation des molécules, un bon procédé est la congélation des échantillons suivie d'un broyage dans l'azote liquide (15).

En règle générale, il faudra conditionner le prélèvement le plus rapidement possible entre le moment de son recueil et son stockage dans des conditions qui préservent la matrice et la substance à analyser.
Lors de la mise au point de la méthode de dosage, il faudra également s'assurer que l'anticoagulant choisi n'a pas d'influence sur les performances de la méthode analytique utilisée pour quantifier le composé $(16,17)$. Récemment, Kummerle et al (18), ont mis en évidence, chez l'animal, que l'héparine influençait le dosage de la doxorubicine.

\section{Élimination des composés interférents}

Les éléments importants à considérer lors de la mise au point du traitement d'un échantillon biologique sont 1) les propriétés physico-chimiques de l'analyte, 2) la nature de la matrice, 3) le recueil de l'échantillon avant l'étape de pré-traitement, 4) la stabilité de l'analyte pendant les différentes étapes du pré-traitement, 5) le solvant utilisé lors de l'étape finale (reprise de l'extrait sec), qui doit être compatible avec le système chromatographique utilisé, 6) le rendement de l'extraction qui doit être le plus élevé et reproductible possible et enfin 7) la reproductibilité, la justesse et l'exactitude de ce traitement.

Avant d'aborder les différentes méthodes de pré-traitement des échantillons en bioanalyse, il faut rappeler qu'il est possible d'injecter directement l'échantillon biologique dans la colonne analytique en CLHP. Les injections directes sont uniquement envisageables pour des liquides biologiques (urine ou liquide céphalorachidien par exemple) contenant de fortes concentrations de l'analyte et de faibles concentrations en protéines. Dans ce cas, la matrice est juste diluée dans de l'eau désionisée avant l'injection. Cette approche est également possible en CG. Une méthode permettant le dosage direct de l'éthanol dans le sang total, le sérum, l'urine et les selles a été décrite par Tangermann (19).

\section{Ultrafiltration et ultracentrifugation}

Seule la fraction libre du médicament est pharmacologiquement active. L'ultrafiltration permet une élimination des protéines par filtration du plasma à travers une membrane semi-perméable. Un certain nombre de kits sont commercialisés à cet usage, parmi les plus utilisés on peut citer les dispositifs «Centrifree ${ }^{\circledast}$ et MPS micropartition ${ }^{\oplus} »$ commercialisés par Amicon $(20,21)$, «Emit Free Level Filtre ${ }^{{ }}$commercialisé par Syva (22) et «Molcut $2^{\oplus}$ » commercialisé par Millipore (23). Seules les petites molécules passent, les protéines sont retenues à la surface du filtre. D'emploi simple et rapide, ils permettent d'éliminer $99 \%$ des protéines. (24). Il y a cependant un certain nombre de sources d'erreur qui sont liées 1) au risque d'adsorption des analytes sur la membrane, en particulier si l'analyte est présent à 
faible concentration, 2) au passage de l'analyte lié à travers la membrane, 3) à l'instabilité de l'équilibre de liaison analyte/protéines durant le processus de séparation, et enfin 4) à la formation d'un dépôt de protéines à la surface de la membrane qui est un facteur limitant de la vitesse et de l'efficacité du processus.

La séparation des composés libres peut également être réalisée par ultracentrifugation. Le plasma est centrifugé à grande vitesse $(100000 \mathrm{~g}, 24 \mathrm{~h})$. Cela permet d'obtenir les protéines et la forme liée de la molécule dans le culot et un surnageant contenant la forme libre. Cependant des erreurs sont possibles dans l'estimation du composé libre dues à certains phénomènes physiques comme la sédimentation, la «back diffusion», la viscosité et la liaison de l'analyte aux lipoprotéines qui surnagent. $(22,23)$.

\section{Précipitation des protéines}

Etant donné leur caractère de zwitterion, les protéines sont chargées positivement en milieu fortement acide et négativement en milieu fortement basique.

Leur précipitation (24) peut être obtenue par modification 1) de la force ionique (utilisation d'une solution saline saturée de sulfate d'ammonium), 2) du pH (ajout en petite quantité d'une solution d'acides forts : acide perchlorique ou trichloracétique essentiellement, ou ajout d'une solution basique toujours en faible volume : sulfate de zinc/hydroxyde de sodium ou sulfate de zinc/hydroxyde de baryum), ou 3) de la constante diélectrique (ajout d'acétonitrile, méthanol, éthanol ou acétone). Apres centrifugation, une partie aliquote du surnageant est injectée dans le système chromatographique. Cependant, le liquide surnageant contient d'autres constituants à coté des protéines et fréquemment le pic de l'analyte peut être accompagné de nombreux autres pics qui sont néfastes pour la spécificité de la méthode.

L'utilisation d'acides forts peut endommager la colonne analytique. Il faut alors utiliser des colonnes spécialement adaptées aux pH très acides $(<1)$ ou ajouter à la phase mobile une forte concentration molaire de tampon. Cette méthode ne peut pas être utilisée pour des composés pouvant subir une hydrolyse acide. Les agents cationiques qui forment des sels insolubles avec les groupements carboxylates des protéines ne sont pas utilisables pour les analytes ayant tendance à former des complexes métalliques.

Les composés cationiques et les acides forts peuvent être utilisés en association avec les solvants organiques (24).

Lorsque la dilution de l'échantillon n'est pas un problème (limite de quantification) des solvants organiques miscibles à l'eau peuvent être utilisés, ils sont moins agressifs que les composés ioniques. L'échantillon est centrifugé pour produire un surnageant clair contenant le composé recherché, et une partie aliquote est injectée dans la colonne. Il est important d'utiliser un solvant dans lequel l'analyte soit hautement soluble, dans le cas contraire il pourrait précipiter avec les protéines. Diluer plus de trois fois le plasma (ou le sérum) avec le solvant organique va entraîner une précipitation de $99 \%$ des protéines (24), mais en contre partie la sensibilité de la méthode va diminuer. Ce problème peut être contourné en augmentant le volume injecté, mais le risque est d'affecter l'efficacité de la chromatographie et la symétrie du pic. Alternativement le surnageant peut être évaporé, mais cette méthode concentre aussi les composés interférents. Enfin un solvant organique non miscible à l'eau, comme le dichlorométhane, peut être ajouté au sumageant. Dans ce dernier cas, la phase inférieure correspond au mélange dichlorométhane-acétonitrile (ou méthanol) et celle supérieure correspond à la phase aqueuse (25-27). Ces dernières peuvent être séparées en utilisant un filtre séparateur de phases en silicone.

Cette méthode présente de nombreux avantages qui sont sa rapidité, sa simplicité et son utilisation pour l'analyse de molécules très hydrosolubles et donc difficilement extractibles. De plus, elle peut être entièrement automatisée comme cela a été décrit pour le salbutamol avant l'analyse de cette molécule par CLHP/MS/MS (28).

Cette méthode est particulièrement bien adaptée au suivi thérapeutique et à la toxicologie.

\section{Démixtion}

La demixtion est basée sur l'élimination des protéines dans l'échantillon, à l'aide d'un solvant organique miscible à l'eau. Dans un premier temps on mélange l'échantillon et le solvant (en général il s'agit de l'acétonitrile) puis on ajoute un sel en excès (carbonate de sodium, chlorure de potassium, ou chlorure de sodium). L'acétonitrile n'est alors plus miscible à l'eau. Après centrifugation, on retrouve du fond du tube vers le haut le sel en excès, la phase aqueuse saturée de sel, le précipité de protéines et enfin la phase organique contenant l'analyte. Cette méthode est particulièrement adaptée au suivi thérapeutique et à la toxicologie. Elle a été utilisé récemment pour le dosage des antiproteases dans le plasma de patients infectés par le virus du SIDA (29). Cette méthode a été également utilisée pour quantifier le fluconazole, l' itraconazole et la lamotrigine dans le plasma (30-32). Il a été récemment publié que le processus de démixtion pouvait être accéléré par l'utilisation d'ultrasons $(33,34)$. En effet, le champ acoustique crée des courants de circulation au sein du mélange, lesquels favorisent le fusionnement 
des gouttelettes, ce qui conduit à une séparation plus rapide des deux phases.

\section{Extraction liquide-liquide}

L'extraction liquide-liquide est connue pour générer des extraits relativement propres avec un bon coefficient d'extraction (35). C'est souvent la méthode de choix pour quantifier de très faibles concentrations d'un composé (de l'ordre du $\mathrm{pg} / \mathrm{ml}$ ). Cependant cette méthode de purification a longtemps été difficilement automatisable ; elle est de plus longue à mettre en œuvre. Récemment, certains auteurs ont développé des méthodes d'extraction liquide-liquide sur plaques 96 puits semi automatisées (36-38) ou entièrement automatisées (39-43).

\section{Extraction liquide-liquide classique}

De part ses avantages et sa facilité d'utilisation, cette méthode reste très largement utilisée pour la préparation des échantillons biologiques. En outre, l'extraction liquide-liquide permet également la concentration de l'analyte. Cette technique est basée sur l'affinité de l'analyte entre une phase aqueuse et une phase organique non miscibles. Le partage d'un composé neutre $i$, entre deux phases non miscibles, est donné par le coefficient de partage $\mathrm{Ki}$ :

$$
\mathrm{Ki}=[\mathrm{Ci}]_{\text {org }} /[\mathrm{Ci}]_{\mathrm{aq}} \text { où }[\mathrm{Ci}]_{\text {org }} \text { et }[\mathrm{Ci}]_{\mathrm{aq}}
$$

sont respectivement les concentrations de l'analyte dans la phase organique et dans la phase aqueuse. $\mathrm{Ce}$ coefficient de partage est constant pour une température donnée, il dépend du solvant organique mais aussi du $\mathrm{pH}$ et de la force ionique de la solution. Il est bien connu que des extractions répétées ( 2 ou plus) avec une faible proportion de 'solvant sont plus efficaces qu'une seule extraction avec un grand volume de solvant (44). Il faut souligner le fait que la multiplication des extractions entraîne la consommation d'une quantité importante de solvant et une perte de temps non négligeable. Aussi, en pratique, un échantillon biologique ne sera pas extrait plus de deux fois.

La polarité est généralement le facteur le plus important dans le choix du solvant organique. En général, les solvants polaires extraient mieux les substances polaires et inversement les solvants apolaires extraient mieux les substances apolaires. La polarité des principaux solvants utilisés est présentée Tableau I. Trois principales interactions entre l'analyte et le solvant d'extraction sont à considérer : 1) les interactions de dispersions (forces de London), 2) les interactions dipôles-dipôles, et 3) la formation de liaisons hydrogènes.

Il faut trouver un bon compromis concernant la polarité du solvant ; ce dernier doit extraire l'analyte avec un coefficient d'extraction maximum mais doit extraire le
Tableau I : Polarité et pouvoir d'élution des principaux solvants.

\begin{tabular}{|c|c|c|c|}
\hline Solvant & Polarité & Pouvoir d'élution & Miscibilité à l'eau \\
\hline Hexane & 0,06 & 0,00 & non \\
Tétrachlorure de carbone & 1,60 & 0,14 & non \\
Ether diéthylique & 2,90 & 0,29 & non \\
Chloroforme & 4,40 & 0,31 & non \\
Chlorure de méthylène & & & \\
ou dichlorométhane & 3,40 & 0,32 & non \\
Acétone & 5,40 & 0,43 & oui \\
Acétate d'éthyle & 4,30 & 0,45 & faible \\
Acétonitrile & 6,20 & 0,50 & oui \\
Isopropanol & 4,3 & 0,63 & oui \\
Méthanol & 6,6 & 0,73 & oui \\
Eau & 10,2 & $>0,73$ & oui \\
\hline
\end{tabular}

moins possible de composés endogènes. Le solvant le plus utilisé est le dichlorométhane qu'il faudra préférer au chloroforme en raison de son potentiel cancérigène. Les analytes très polaires sont difficiles à extraire et nécessitent l'utilisation de solvants très polaires et par conséquent non sélectifs. Il est possible alors de faire appel à des mélanges de solvants (le plus souvent binaires). L'association de 1 à $5 \%$ d'un alcool (par exemple l'isopropanol) et d'un solvant peu polaire (par exemple le dichlorométhane) est la plus fréquente. Ceci va avoir pour effet d'éviter l'adsorption de l'analyte sur le verre (particulièrement si celui-ci est présent à faible concentration), d'augmenter le coefficient d'extraction, mais aussi de permettre l'extraction simultanée de la molécule parente et de ses métabolites (souvent plus polaires).

Un article de synthèse concernant les méthodes d'extraction en toxicologie a récemment été publié par Drummer (45). Parmi les solvants organiques les plus utilisés, cette auteur rapporte l'acétate d'éthyle, le mélange dichlorométhane-acétone, le mélange toluèneacétate d'éthyle et le mélange dichorométhane-isopropanol-acétate d'éthyle.

Seule la forme non ionisée de l'analyte est extraite dans le solvant organique. Le $\mathrm{pH}$ optimal d'extraction des composés acides est de 1 à 2 unités en dessous des valeurs du pKa. ; il est de 1 à 2 unités de $\mathrm{pH}$ au dessus des valeurs du pKa pour les composés basiques. Mais l'extraction peut être difficile pour les composés qui sont solubles dans l'eau à toutes les valeurs de $\mathrm{pH}$, par exemple les composés amphotères ou neutres, avec lesquels on ne pourra pas jouer sur des variations de $\mathrm{pH}$. Dans certains cas l'addition de solutions tampons à la phase aqueuse augmente sa force ionique et par conséquent sa polarité ce qui va diminuer l'affinité du composé pour la phase aqueuse et favoriser son passage dans la phase organique. 


\section{Double extraction liquide-liquide}

Dans le but d'augmenter la pureté des extraits, une préextraction des composés interférents de la matrice peut être réalisée (44). Par exemple, l'urine contient de nombreux composés endogènes; une extraction préliminaire de l'urine en milieu acide améliore la pureté de l'extrait obtenu après alcalinisation. Une autre méthode très utilisée est une première extraction en milieu alcalin (l'analyte passe dans la phase organique) suivie d'une deuxième extraction en milieu acide (l'analyte sera sous forme ionisée donc non extractible par le solvant organique). Pour les composés basiques, on préférera utiliser l'acide sulfurique ou l'acide phosphorique plutôt que l'acide chlorhydrique car beaucoup de chlorures sont solubles dans les solvants organiques. La double extraction liquide lquide est plus ou moins obligatoire en GC puisque les substances endogènes issues de la matrice tendent à contaminer les détecteurs, particulièrement le détecteur à capture d'électrons. De même, cette approche est largement utilisée en CLHP. La double extraction augmente considérablement la pureté de l'analyse.

\section{Extraction par formation de paires d'ions}

Cette méthode est utilisée pour extraire des composés chargés, type ammoniums quaternaires, très polaires et donc solubles dans l'eau. Le principe est d'ajouter à la phase aqueuse un excès d'un "contre-ion» volumineux hydrophobe et de charge opposée, afin de masquer la charge de l'ion à extraire (Figure 1). L'ensemble forme une espèce pseudo-moléculaire électriquement neutre ou «paire d'ions», extractible par un solvant organique.

Parmi les contre-ions les plus utilisés, il faut citer les acides sulfoniques et le dodecylsulfate de sodium pour les composés basiques et les bromures ou hydroxides de tetra (méthyl, éthyl, propyl, butyl ou pentyl) ammonium pour les composés acides. La formation de la paire d'ions dépend du $\mathrm{pH}$ de la phase aqueuse, du type de solvant organique utilisé, ainsi que de la nature et de la concentration du contre-ion. La technique d'extraction par formation de paire d'ions est utilisable pour une variété de composés ionisables qui sont difficiles à extraire sous leur forme inchangée comme les pénicillines, les aminoacides et les métabolites conjugués. Cette méthode permet l'extraction d'ammonium quaternaires comme la tubocurarine qui est ionisée à toutes les valeurs de $\mathrm{pH}$. Dans certain cas, en CLHP, le contreion peut être ajouté à la phase mobile pour réduire le temps de rétention des solutés. Par exemple, les temps de rétention de l'énoxacine, de son métabolite, la 4oxo-enoxacine, et de la péfloxacine diminuent lorsque la concentration d'hydroxyde de tétrabutylammonium augmente dans la phase mobile ; les pics deviennent alors plus fins et plus symétriques suite à la diminution du temps de rétention (15).

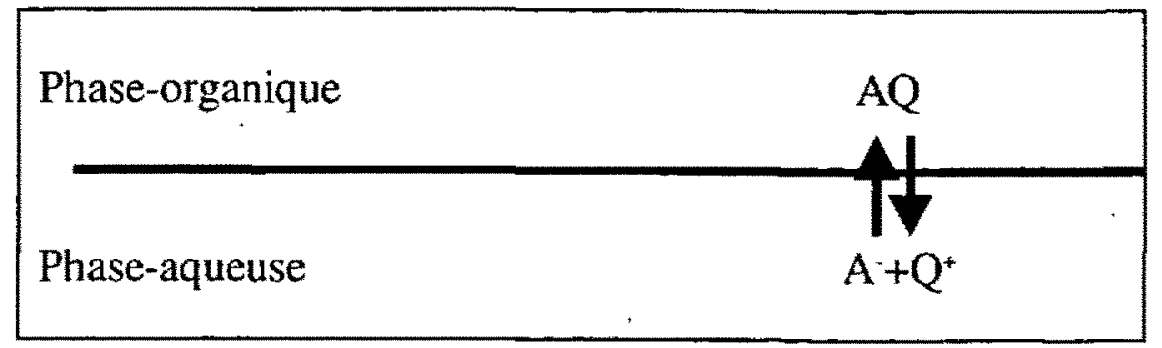

Figure 1 : Principe de l'extraction par formation de paires d'ions.

\section{Extraction par solvant après dérivation}

C'est la CG qui a profité le plus de telles méthodes de transformations chimiques. Une méthode par $\mathrm{CG}$ et détection par capture d'électron (46) a été développée pour le dosage de 26 benzodiazépines et de leurs métabolites dans le sang total. Les analytes sont extraits de la matrice par l'acétate d'éthyle après dérivation. Les auteurs ont utilisé cette méthode pour la recherche et le dosage de ces composés dans du sang de cadavre. Récemment, Pirnay et al. (47) ont développé un méthode par CG/MS permettant l'analyse simultanée de 22 benzodiazépines dans le sang et l'urine en vue d'une utilisation en toxicologie médico-légale. La dérivation en CLHP n'est pas utilisée pour les mêmes raisons qu'en CG. Elle permet principalement 1) d'améliorer la détection des analytes, bien que dans certains cas, elle soit nécessaire pour libérer les analytes de leurs sites de liaison, 2) d'augmenter la résolution de composés très proches (racémates), 3) de confirmer l'identité d'un analyte, 4) d'augmenter la sélectivité d'une analyse dans une matrice complexe et 5) d'améliorer le comportement chromatographique du composé étudié. On distingue plusieurs types de dérivations suivant qu'elles seront effectuées avant ou après la colonne chromatographique, mais aussi suivant qu'elles sont effectuées en ligne ou hors ligne. Par exemple, l'agent anti-néoplasique cisplatine est chélaté par le diethyldithiocarbamate pour former un sel absorbant dans l'ultraviolet avant l'analyse par CLHP. Le dosage est réalisé en présence de sel de nickel comme étalon interne qui se dérive dans les mêmes conditions (48).

\section{Extraction solide-liquide hors ligne}

Le principe de l'extraction solide-liquide est la rétention sélective du ou des analytes sur une phase stationnaire grâce à des interactions intermoléculaires entre l'analyte et les groupements fonctionnels de la phase stationnaire. Ces interactions sont de type ionique (première forces impliquées dans l'extraction par échange d'ions), liaisons hydrogène, interactions dipôle-dipôle et liaisons de Van der Waals.

Les différentes étapes de l'extraction solide-liquide (49) sont présentées Tableau II.

De nombreux supports sont actuellement disponibles permettant l'extraction de pratiquement toutes les classes de composés $(45,50)$. 
Tableau II : Extraction solide-liquide hors ligne : étape 1 ; pré-traitement de l'échantillon; étape 2 : conditionnement de la colonne; étape 3 : équilibrage de la colonne.

\begin{tabular}{|c|c|c|c|}
\hline Étapes & But & Méthodes utilisées & Particularités \\
\hline 1 & $\begin{array}{l}\text { Modifier } \\
\text { l'échantillon tant } \\
\text { d'un point de vu } \\
\text { physique que } \\
\text { chimique. }\end{array}$ & $\begin{array}{l}\text { - Ajustement du } \mathrm{pH}^{\mathrm{a}} \\
\text { - Utilisation d'agents mouillants } \\
\text { (ex :méthanol) } \\
\text { - Dilution }\end{array}$ & $\begin{array}{l}\text { Étape conditionnée par la stabilité } \\
\text { de l'analyte, le type de matrice, le } \\
\text { type de la phase stationnaire et par } \\
\text { l'interaction analyte/phase } \\
\text { stationnaire. }\end{array}$ \\
\hline 2 & $\begin{array}{l}\text { Activation de } \\
\text { l'adsorbant }\end{array}$ & $\begin{array}{l}\text { - Si échantillon aqueux, utilisation } \\
\text { d'un solvant organique très polaire } \\
\text { miscible à l'eau (généralement } \\
\text { méthanol) } \\
\text { - Si échantillon non-aqueux } \\
\text { utilisation de la matrice elle même. }\end{array}$ & $\begin{array}{l}\text { Ordre de grandeur du volume de } \\
\text { solvant à utiliser : } 2 \text { fois celui du } \\
\text { support. }\end{array}$ \\
\hline 3 & $\begin{array}{l}\text { Éliminer l'excès de } \\
\text { solvant d'activation }\end{array}$ & $\begin{array}{l}\text { Pour les échantillons en solution } \\
\text { aqueuse, utiliser une solution dont le } \\
\text { pH et la force ionique sont } \\
\text { similaires à ceux de la matrice }\end{array}$ & $\begin{array}{l}\text { Étape nécessaire si l'échantillon } \\
\text { est aqueux mais inutile si } \\
\text { l'échantillon est non- aqueux }{ }^{b}\end{array}$ \\
\hline
\end{tabular}

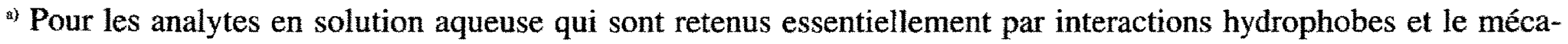
nisme de rétention de l'analyte est l'échange d'ions.

b) Généralement le support ne doit pas sécher entre les étapes 2 et 3 et l'ajout de l'échantillon à analyser. Actuellement il existe dans le commerce des colonnes pour lesquelles les étapes 2 et 3 ne sont pas nécessaires.

Tableau II (suite) : Extraction solide-liquide hors ligne : étape 4 : chargement de l'échantillon sur la colonne ;étape 5 : élimination des composés interférents; étape 6 : élution des analytes.

\begin{tabular}{|c|c|c|c|}
\hline Étapes & But & Méthodes utilisées & Particularités \\
\hline 4 & $\begin{array}{l}\text { Optimiser le débit } \\
\text { pour rétention } \\
\text { maximale de } \\
\text { l'analyte sur la } \\
\text { phase stationnaire }\end{array}$ & $\begin{array}{l}\text { Généralement } 0,5 \text { à } 1 \mathrm{ml} \\
\text { d'échantillon pour une colonne } \\
\text { de } 100 \mathrm{mg} \text {. }\end{array}$ & $\begin{array}{l}\text { - Nécessiter de travailler à débit } \\
\text { constant. } \\
\text { - Commencer à débit faible } 1 \mathrm{ml} / \mathrm{min} \\
\text { si cartouche de } 1 \mathrm{ml}, 3 \mathrm{ml} / \mathrm{min} \text { si } \\
\text { cartouche de } 3 \mathrm{ml}, 7 \mathrm{ml} / \mathrm{min} \text { si } \\
\text { cartouche de } 6 \mathrm{ml} \text { puis augmenter. }\end{array}$ \\
\hline 5 & $\begin{array}{l}\text { Eliminer les } \\
\text { composés } \\
\text { interférents en } \\
\text { évitant la perte } \\
\text { d'analyte. }\end{array}$ & $\begin{array}{l}\text { - Utilisation d'un liquide de lavage } \\
\text { miscible avec la matrice ( } 1 \text { à } 2 \mathrm{ml} \\
\text { pour } 100 \mathrm{mg} \text { de phase stationnaire) } \\
\text { - Si liquide de lavage non miscible à } \\
\text { l'eau, sécher la cartouche avant } \\
\text { l'étape de lavage }\end{array}$ & $\begin{array}{l}\text { Si échantillon aqueux, nécessité de } \\
\text { maintenir } \mathrm{pH} \text { et force ionique } \\
\text { constants. }\end{array}$ \\
\hline 6 & $\begin{array}{l}\text { Travailler dans des } \\
\text { conditions optimales } \\
\text { d'élution. }\end{array}$ & $\begin{array}{l}\text { - Débit de solvant d'élution constant. } \\
\text { - L'analyte doit être soluble dans le } \\
\text { solvant d'élution. }^{c}\end{array}$ & $\begin{array}{l}\text { Plusieurs petits volumes sont plus } \\
\text { efficaces qu'un grand volume. }\end{array}$ \\
\hline
\end{tabular}

${ }^{\text {c) }}$ si les analytes sont retenus par échange d'ions, il faut utiliser des tampons de force ionique élevée (concentration molaire $>0,1 \mathrm{M}$ ), si l'échantillon possède 2 charges, la concentration du tampon devra être $>0,2 \mathrm{M}$. Un composant organique dans le liquide d'élution peut être nécessaire afin d'éviter des interactions hydrophobes secondaires. 


\section{Utilisation de phases stationnaires non greffées polaires}

La silice, le kieselguhr (particules inertes de terre de diatomée) et l'alumine ont été les premières phases stationnaires à être utilisées pour la séparation de composés polaires (51-53). Le Florisil ${ }^{\circledR}$ (silicate de magnésium activé) est encore très utilisé pour l'isolement des pesticides. Ces phases stationnaires ont des propriétés adsorbantes. La silice est la phase stationnaire non greffée la plus couramment utilisée. Trois sites participent au mécanisme de rétention : 1) les silanols libres $(\equiv \mathrm{SiOH}), 2)$ les silanols liés par liaisons hydrogènes et 3) les silanols libres recouverts par une molécule d'eau. Les groupements siloxane ( $\equiv \mathrm{Si}-\mathrm{O}-\mathrm{Si} \equiv$ ) obtenus par déshydratation de 2 silanols voisins n' interviennent pas dans la chromatographie. Les sites polaires sur la silice adsorbent modérément les composés polaires dissous dans des solvants organiques ayant un pouvoir d'élution inférieur à environ 0,38 (Tableau I). Les alcools, les aldéhydes dissous dans un solvant non polaire sont bien adsorbés. Ces analytes peuvent être élués de la silice par des solvants ayant des forces d'élution supérieures à environ 0,6 (Tableau I). Ces solvants forment des liaisons hydrogènes fortes avec les groupements silanols déplaçant ainsi l'analyte. En général les composés basiques sont fortement retenus sur la silice et les composés acides sont fortement retenus sur l'alumine.

\section{Utilisation de phases stationnaires de silice gref- fée polaires}

Les phases stationnaires ainsi utilisées sont celles possédant des groupements cyano, aminopropyl ou diol .

L'échantillon à analyser est dissout dans un solvant non-polaire (par exemple l'hexane). Lors du passage à travers la colonne, l'analyte est adsorbé sur la phase stationnaire (interactions dipôles). Il est ensuite élué (formation de liaisons hydrogènes avec l'analyte) par un solvant très polaire (par exemple le méthanol) $(45$, 54-56).

\section{Utilisation de phases stationnaires de silice gref- fée non-polaires}

Un mécanisme de partage à polarité inversée de phases est mis en jeu. La phase stationnaire est moins polaire que la solution de l'échantillon à analyser. Ces colonnes possèdent des groupements siloxanes substitués par des groupements octadecyl, octyl, hexyl, butyl, ethyl, cyclohexyl, phenyl ou cyanopropyl $(45,54-59)$. Après fixation de l'analyte sur la colonne à partir d'une matrice aqueuse polaire, ce dernier est élué par un solvant organique. Ce solvant organique peut être du tétrahydrofurane (force éluante 4,4), de l'isopropanol (force éluante 4,2), de l'éthanol (force éluante 3,6), du dioxane (force éluante 3,5), de l'acétone (force éluante 3,4 ), de l'acétonitrile (force éluante 3,1) ou du métha- nol (force éluante 3,0). Ce sont les colonnes possédant des groupements octadecyl qui ont le pouvoir de rétention le plus élevé. Ce pouvoir de rétention varie dans l'ordre: $\mathrm{C}_{18}>\mathrm{C}_{8}>\mathrm{C}_{6}>\mathrm{C}_{4}>\mathrm{C}_{2}$ (si interactions nonpolaires uniquement).

Les interactions entre analytes et phases stationnaires sont des forces intermoléculaires essentiellement 1) liaisons de van der Waals, 2) interactions dipôle-dipôle et 3) liaisons hydrogène.

\section{Utilisation de colonnes de silice greffée échan- geuses d'ions}

Ces phases sont utilisées pour l'extraction de composés acides ou basiques à partir d'une solution aqueuse (60). Les phases stationnaires les plus fréquemment utilisées sont celles contenant les groupements fonctionnels ioniques suivants : $-\mathrm{SO}_{3}{ }^{-}$(échangeur fort de cations : acide benzène sulfonique, acide propyl sulfonique et acide éthyl benzène sulfonique) pour l'extraction de composés basiques et triéthylaminopropyl $\left(-\left(\mathrm{CH}_{2}\right)_{3} \mathrm{~N}^{+}\left(\mathrm{CH}_{3}\right)_{3}\right.$, échangeur fort d'anions) pour l'adsorption de composés acides. Il existe également des échangeurs faibles d'anions (contenant les groupements $-\left(\mathrm{CH}_{2}\right)_{3} \mathrm{NH}_{2}$ ou $\left.-\left(\mathrm{CH}_{2}\right)_{3} \mathrm{NH}\left(\mathrm{CH}_{2}\right)_{2} \mathrm{NH}_{2}\right)$ et de cations (contenant le groupement $\left.-\left(\mathrm{CH}_{2}\right)_{3} \mathrm{COOH}\right)$. Le principe est le suivant, 1) échange entre les composés ionisés à analyser et le contre-ion de charge identique de la phase stationnaire et 2) élution des composés à analyser par une modification du $\mathrm{pH}$ du solvant d'élution ( $\mathrm{pH}=\mathrm{pK}_{\mathrm{a}} \pm 1$ ou 2 unités de $\mathrm{pH}$ ) entraînant ainsi le changement de la charge du composé ou par un solvant d'élution contenant un contre-ion compétitif de même signe, mais d'affinité plus grande pour l'ion de la phase stationnaire.

Les différents facteurs affectant la sélectivité de l'échange d'ions sont : 1) le $\mathrm{pH}, 2$ ) la sélectivité du contre ion. 3) la force ionique (une force ionique faible favorise la rétention tandis qu'une force ionique élevée favorise l'élution); et 4) le débit de la solution à travers le support (les interactions par échange d'ion se produisent à un débit inférieur à celui des interactions polaires ou non-polaires). Pour les cations, la sélectivité (déplacement de la droite vers la gauche) est la suivante : $\mathrm{Li}^{+}<\mathrm{H}^{+}<\mathrm{Na}^{+}<\mathrm{NH}_{4}{ }^{+}<\mathrm{RNH}_{3}{ }^{+}<\mathrm{K}^{+}<\mathrm{Mg}^{2+}<$ $\mathrm{Ca}^{2+}$; pour les anions (déplacement de la droite vers la gauche) elle est : $\mathrm{OH}<$ acétate $<$ formiate $<\mathrm{HCO}_{3}{ }^{\circ}<$ $\mathrm{Cl}^{-}<\mathrm{HSO}_{3}<\mathrm{CN}<$ citrate $<$ benzène sulfonate.

\section{Remarque concernant l'utilisation des colonnes de silice greffée}

Les silices greffées sont obtenues par réaction des groupements silanols libres de la silice avec des agents dérivatisants mono- ou trifonctionnel. Cependant, il reste toujours quelques silanols libres après la réaction de dérivatisation. Par conséquent, ces colonnes présen- 
tent des propriétés hétérogènes dues d'une part aux silanols libres et d'autre part aux silanols greffés. Ces silanols libres peuvent être désactivés par masquage avec du triméthylchlorosilane $\left(\mathrm{Cl}-\mathrm{Si}\left(\mathrm{CH}_{3}\right)_{3}\right)$.

\section{Utilisation de phases stationnaires de silice gref- fée en mode mixte}

Ces phases contiennent des groupements fonctionnels non-polaires $\left(\mathrm{C}_{18}, \mathrm{C}_{8}\right.$ ou $\left.\mathrm{C}_{4}\right)$ et des groupements échangeurs forts de cation ou d'anion $(51,61-64)$.

L'utilisation de telles colonnes permet d'obtenir des extraits particulièrement purs. Ces colonnes sont très utilisées pour l'analyse des stupéfiants. C'est ainsi que les colonnes mixtes ayant les groupements fonctionnels $\mathrm{C}_{8}$ et $-\mathrm{NR}_{3}{ }^{+}$sont particulièrement bien adaptées à l'extraction de l'acide carboxylique du 11 Nor- $\Delta$-9-tetrahydrocanabinol (métabolite majeur du cannabis) à partir de l'urine. Récemment, Gustafson et al. (61) proposent une méthode de dosage du $\Delta-9$-tetrahydrocanabinol (THC), du 11-hydroxy-THC et du 11-nor-9-carboxy-THC dans le plasma. Les analytes sont séparés de la matrice biologique par extraction solide-liquide sur des colonnes mixtes Clean Screen (Bristol technology, PA).

Le tableau III regroupe les phases stationnaires spécifiques développées pour les 5 classes de stupéfiants listés par la SAMHSA (Substance Abuse and Mental Health Service Administration), ex NIDA (National Institute of Drug Abuse).

Tableau III : Phases stationnaires mixtes développées pour les 5 classes de stupéfiants.

\begin{tabular}{|c|c|}
\hline \multicolumn{2}{|c|}{ 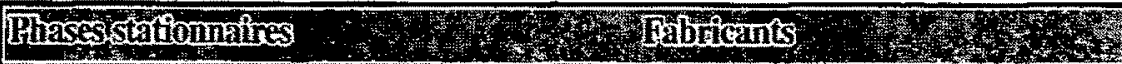 } \\
\hline \multicolumn{2}{|c|}{$\begin{array}{l}\text { Bond-Elut Certify I } \mathrm{C}_{8} / \mathrm{EC} \\
\text { et II } \mathrm{C}_{8} / \mathrm{EA}\end{array}$} \\
\hline \multicolumn{2}{|r|}{ United Chemical Technologies } \\
\hline \multirow{2}{*}{\multicolumn{2}{|c|}{$\begin{array}{l}\text { Narc-1 (methylester et Narc-2 (cation / HIC) } \\
\text { Isolute-Confirm } \mathrm{HAX}: \mathrm{C}_{8} / \mathrm{EA} \\
\qquad \mathrm{HCX}: \mathrm{C}_{18} \text { ou } \mathrm{C}_{4} / \mathrm{EA}\end{array}$}} \\
\hline & \\
\hline \multirow{2}{*}{$\begin{array}{l}\text { Evidex (polypropylène) } \\
\text { Spec }\left(\mathrm{C}_{18} / \mathrm{EC} \text {; Phase peu polaire / EC) }\right.\end{array}$} & $J \& W$ Scientific \\
\hline & Ansys Diagnostics \\
\hline
\end{tabular}

$E C$, échangeur de cations ; EA, échangeur d'anions ; EI, échangeur d'ions ; $\mathrm{HIC}$, "hydrophobic interaction chromatography"

\section{Phases stationnaires basées sur un mode d'ex- clusion}

Des phases stationnaires avec des diamètres de pores contrôlés sont disponibles. Les molécules de faible masse moléculaires $(<10000 \mathrm{D})$ sont retenues alors que les grosses molécules sont éluées.

\section{Autres phases stationnaires}

Des phases stationnaires à base de copolymère sont également disponibles. Il s'agit de copolymère hydro- phile-lipophile (polystyrène-divinylbenzène) hydroxylé (ne nécessitant pas de conditionnement avant son utilisation) ou non modifié (doit être conditionné avant son utilisation) (51). Ces colonnes sont idéales pour l'extraction de composés polaires qui ne sont pas adéquatement retenus sur des phases stationnaires $\mathrm{C}_{18}$ ou $\mathrm{C}_{8}$. Les avantages de ces phases stationnaires par rapport aux silices greffées sont 1) une structure très homogène (plus de problème de silanols libres), 2) une reproductibilité de fabrication, 3) une stabilité entre $\mathrm{pH}$ 1 et 14 au lieu de 2 et 7 pour les silices utilisées en chromatographie de paires d'ions, et 4) bien qu'étant une phase stationnaire apolaire, la structure aromatique du polymère met en jeu des interactions (type Van der Waals) par les électrons d'où un mode de rétention différent de celui des silices greffées. Les inconvénients sont les suivants : résistance à la pression très inférieure (problème en CLHP) et transfert de masse plus lent. Les phases stationnaires à base de copolymère de polystyrène-divinylbenzène non modifié ont été utilisées pour le screening toxicologique (même pour des milieux complexes comme le sang post-mortem ou les tissus) à cause du caractère non sélectif de l'adsorbant (65).

Des phases stationnaires à base de copolymère de $\mathrm{N}$ vinylpyrrolidone et divinylbenzene sont également disponibles. Ces colonnes ont une grande capacité de rétention et peuvent être utilisées, entre $\mathrm{pH} 1$ et 14 , pour l'extraction de composés lipophiles ou hydrophiles.

\section{Systèmes d'extraction solide-liquide commercia- lisés}

Les systèmes d'extraction solide-liquide les plus utilisés sont les cartouches seringues et les disques. Ces derniers permettent de hauts débits du fait de la combinaison d'une large surface de travail et d'une faible épaisseur de phase stationnaire. Plusieurs variétés de disques sont disponibles commercialement. Les deux types les plus utilisés sont les disques de polytétrafluoroéthylène $\left(\right.$ Teflon $\left.^{\circledR}\right)$ imprégnés de phase et les disques à support en fibre de verre.

Récemment des plaques de 96 puits basées sur une extraction solide-liquide sont apparues sur le marché. Elles permettent un pré-traitement de l'échantillons à haut débit. Ces plaques sont très utilisées lorsque la méthode d'analyse choisie est le couplage CLHP/SM/SM $(38,43,66,67)$.

\section{Extraction solide-liquide en ligne}

Le principe de l'extraction solide-liquide en ligne est présenté figure 2 . La pompe 3 est utilisée pour délivrer la phase mobile et la pompe 1 pour délivrer le solvant de lavage (eau ou tampon avec ou non un faible pour- 


\section{Position 1}

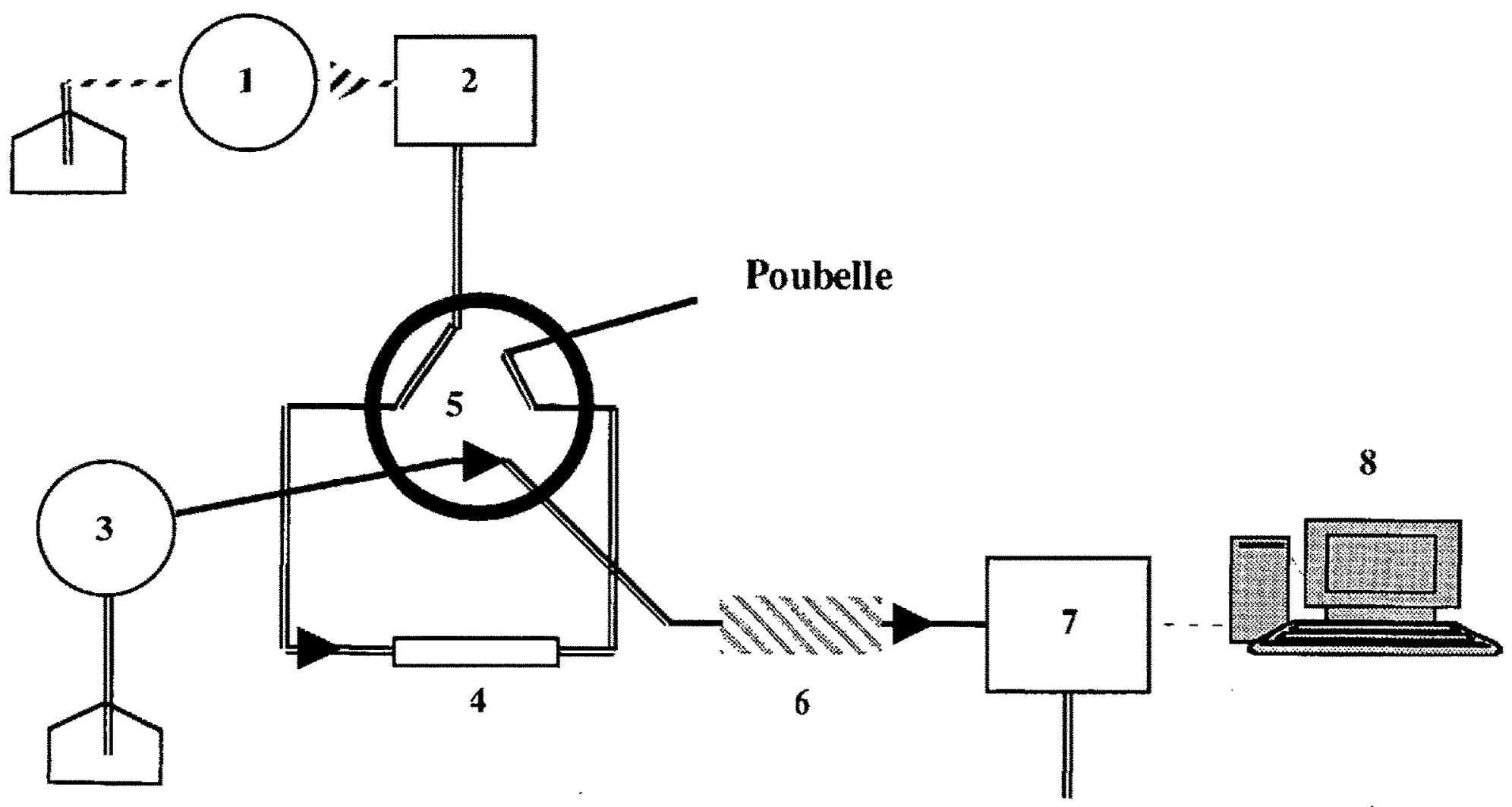

\section{Position 2}

\section{Poubelle}

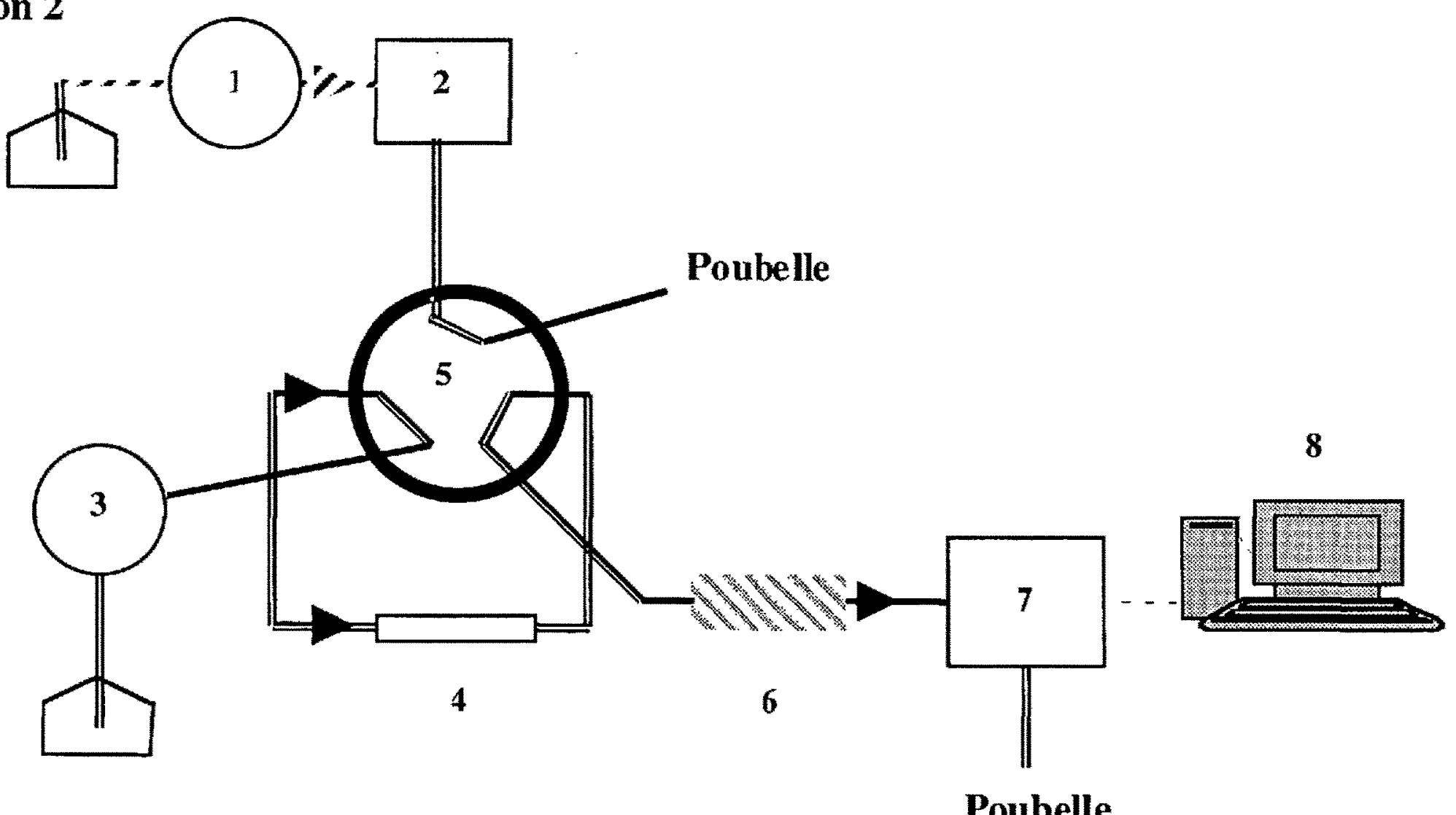

Figure 2 : Principe de l'extraction solide-liquide en ligne 1 et 3 , pompes ; 2 , passeur d'échantillons ; 4, pré-colonne d'extraction solide-liquide en ligne $; 5$, vanne d'injection ; 6 , colonne analytique $; 7$, détecteur $; 8$, système informatique.

centage de solvant organique). En position 1, la vanne d'injection 5 est positionnée de façon à ce que le solvant de lavage circule dans la pré-colonne de concentration grâce à la pompe 1 et que la phase mobile circule dans la colonne analytique grâce à la pompe 3 . L'échantillon biologique est ensuite injecté dans cette pré-colonne. Après l'étape de lavage (élimination des constituants polaires de la matrice grâce au solvant de lavage), le ou les analytes sont concentrés en tête de colonne. La vanne d'injection 5 permute en position 2 ; la pré-colonne de concentration est alors connectée à la colonne analytique. Les analytes sont entraînés dans la colonne analytique par la phase mobile. Après quelques minutes, la vanne d'injection commute en position 1, la pré-colonne est alors rééquilibrée avec le solvant de lavage avant l'injection d'un nouvel échantillon. Les milieux biologiques riches en protéines nécessitent souvent une précipitation préalable des protéines avant l'injection sur la colonne de «switching». Il faut noter que des pré-colonnes basées sur le princi- 
pe de l'exclusion de taille sont disponibles et permettent l'injection directe de ces milieux sur la pré-colonne (68-72).

Cette technique a été utilisée pour doser le midazolam et pour identifier ses métabolites dans le sang et les urines d'un cadavre après un suicide (73). Récemment, Hayashida et al.(74) ont décrit une méthode d'extraction solide-liquide en ligne permettant le dosage de la tétrodotoxine dans le sérum pour une application en toxicologie et en médecine-légale.

\section{Micro-extraction en phase solide (MEPS)}

La MEPS (75-77) ne nécessite ni solvant ni appareillage compliqué. Elle permet de concentrer les composés volatils ou non-volatils présents dans des échantillons avant l'analyse par CG ou CLHP. Les analytes sont adsorbés sur une fibre de silice fondue imprégnée d'une phase stationnaire (Carbowax, polyacrylate ou polydiméthylsiloxane). Les analytes sont désorbés de la fibre par introduction directe dans l'injecteur CG amené à haute température dans le cas d'une analyse par CG ou par un interface SPME/CLHP dans le cas d'une analyse par CLHP. Cette interface extrait les analytes de la fibre de SPME à l'aide d'un solvant qui transporte les analytes désorbés vers la colonne analytique.

La MEPS $(78,79)$ est utilisée en médecine légale et toxicologie pour doser les amphétamines (80-83), les anesthésiques (84), les anti-dépresseurs (85-88), les barbituriques (89), les benzodiazépines (90-91), les opiacés et la cocaïne $(92,93)$, le THC et les autres cannabinoides (92), les pesticides (94) et des substances volatiles (95-98).

\section{Sources d'erreur lors du pré-traitement des échan- tillons}

Si le pré-traitement précède une analyse quantitative, il est nécessaire que l'incertitude due à la méthode d'extraction soit limitée. Il faudra en outre que le partage des composés à quantifier ne soit pas concentrationdépendent (dans le domaine des concentrations choisies).

Le coefficient de partage varie avec la température; il est donc recommandé de travailler à une température contrôlée, l'utilisation d'un étalon interne ne corrigeant que partiellement ce phénomène.

Lors de l'extraction liquide-liquide, la durée et la méthode d'agitation jouent aussi un rôle important pour parvenir à l'équilibre des deux phases. Il est donc important d'optimiser la procédure d'extraction.

Lorsque le solvant organique contenant l'analyte est évaporé sous courant d'azote à des fins de concentration, il y a un risque non négligeable de dégradation des composés instables. Des pertes peuvent aussi se produirent lors de l'étape de re-dissolution et entraîner une diminution du rendement d'extraction. Un autre problème est le risque d'adsorption des analytes sur le verre au cours des étapes d'extraction ou d'évaporation du solvant, surtout lorsque les concentrations sont faibles. Ce problème peut être partiellement surmonté par 1) la silanisation des parois en verre, 2) l'addition de 1 à $2 \%$ d'un adjuvant très polaire (par exemple : l'éthanol, l'isopropanol, ou l'alcool isoamylique) au solvant d'extraction, ou 3) l'utilisation de tubes en polypropylène.

\section{Discussion et conclusion}

Au cours de la procédure de validation d'une méthode de dosage, pour obtenir des résultats précis et fiables, il faut analyser des échantillons relativement «propres». De ce fait, le pré-traitement de l'échantillon biologique est une partie essentielle de toute méthode analytique. Il permet: 1) d'augmenter la durée de vie de la colonne analytique par injection d'extraits contenant un minimum de produits endogènes de la matrice ,2) d'améliorer la reproductibilité de l'analyse, 3) d'abaisser la limite de quantification de la méthode par diminution du bruit de fond et par une étape de concentration, 4) d'améliorer la fidélité et l'exactitude de l'analyse et enfin 5) d'augmenter la sélectivité.

L'analyse quantitative d'un composé peut être erronée lorsque des substances endogènes de la matrice biologique co-éluent avec le composé à doser. Ainsi, la sélectivité de l'analyse doit être évaluée durant le développement et la validation d'une méthode de dosage en testant différentes sources de matrice. Il ne doit pas y avoir de pic du à la matrice au temps de rétention des différents analytes.

La technique de chromatographie liquide haute performance couplée à la spectrométrie de masse (CLHP/SM et CLHP/SM/SM.) est particulièrement sensibles à l'effet matrice $(5,6)$. Cet effet se traduit par un augmentation ou une diminution du signal. Le pré-traitement de l'échantillon doit permettre d'éliminer cet effet.

Le choix de la méthode utilisée sera fonction de différents facteurs. A côté des paramètres physico-chimiques des molécules à quantifier et de la complexité de l'échantillon biologique interviendront la méthode d'analyse et le système de détection choisi. 
L'extraction liquide-liquide est une méthode très sélective, elle a été très utilisée pour l'extraction des médicaments préalablement à l'analyse par CG dans le cas d'un dépistage systématique en toxicologie (45). Son avantage est la possibilité d'obtenir des extraits très propres. L'inconvénient de cette méthode est l'utilisation de volumes importants de solvant organique, la longueur de l'analyse et pendant longtemps sa difficulté d'automatisation. L'extraction solide-liquide est de plus en plus utilisée. Ses avantages sont la diversité des phases stationnaires permettant l'extraction de pratiquement toutes les classes de composés, ses possibilités d'automatisation, ainsi qu'un gain de temps et l'utilisation d'une quantité moindre d'échantillon biologique et de solvant organique par rapport à l'extraction liquide-liquide. Mais la purification et la sélectivité peuvent être limitées; de plus, c'est un matériel cher à automatiser.

\section{Références}

1. Kataoka $\mathrm{H}$. New trends in sample preparation for clinical and pharmaceutical analysis. Trends Anal. Chem. $2003 ; 22: 232-44$.

2. Misl'anová C., Hutta M. Role of biological matrices during the analysis of chiral drugs by liquid chromatography. J. Chromatogr. B Analyst Technol. Biomed. Life Sci. $2003 ; 797: 91-109$.

3. Maurer H.H. Systematic toxicological analysis procedures for acidic drugs and/or metabolites relevant to clinical and forensic toxicology and/or doping control. $J$. Chromatogr. B Biomed. Sci. Appl. 1999 ; 733 : 3-25.

4. Sarkioja T., Yla-Herttuala S., Solakivi T., Nikkari .T., Hirvonen J. Stability of plasma total cholesterol, triglycerides, and apolipoproteins B and A-I during the early postmortem period. J. Forensic Sci. 1988; 33 : 1432-8.

5. Buhrman D.L., Price P., Rudewicz P.J. Quantitation of CR27417 in human plasma using electrospray liquid chromatography tandem mass spectrometry: a study of ion suppression. J. Am. Soc. Mass Spectrom. 1996; 7 : 1099-105.

6. King R., Bonfiglio R., Fernandez-Metzler C., MillerStein $C$., Olah T. Mechanistic investigation of ionization suppression in electrospray ionization. J. Am. Soc. Mass Spectrom. $2000 ; 11: 942-50$.

7. Dams R., Huestis M.A., Lambert W.E., Murphy C.M. Matrix effect in bio-analysis of illicit drugs with LC-MS : influence of ionization type, sample preparation, and biofluid. J. Am. Soc. Mass Spectrom.. 2003 ; 14 : 1290-4.

8. Ostheimer D., Cremese M., Wu A.H., Hill D.W. Reduction in extraction efficiency of charged particles from the ion source as the cause of matrix effects in the GC-MS analysis of drugs. J. Anal. Toxicol. $1997 ; 21$ : 17-22.

9. Shaw J.C. Nonmetabolizable base balance: effect of diet composition on plasma pH. J. Nutr. $1989 ; 119: 1789-98$.
10. Fura A., Harper T.W., Zhang H., Fung L., Shyu W.C. Shift in $\mathrm{pH}$ of biological fluids during storage and processing: effect on bioanalysis. J. Pharm. Biomed. Anal. $2003 ; 32: 513-22$.

11. Paxton J.W. Protein binding of methotrexate in sera from normal human beings: effect of drug concentration, $\mathrm{pH}$, temperature, and storage. J. Pharmacol. Methods. 1981 ; $5: 203-13$.

12. Yeung C.Y., Lee F.T., Wong H.N. Effect of serum pH on bilirubin-protein binding. Acta Paediatr. Jpn. 1992; 34 : 23-7.

13. Brors O., Jacobsen S. pH lability in serum during equilibrium dialysis. Br. J. Clin. Pharmacol. $1985 ; 20: 85-8$.

14. Kearney A.S., Crawford L.F., Mehta S.C., Radebaugh G.W. The interconversion kinetics, equilibrium, and solubilities of the lactone and hydroxyacid forms of the HMG-CoA reductase inhibitor, CI-981. Pharm. Res. $1993 ; 10: 1461-5$.

15. Hamel B., Audran M., Costa P., Bressolle F. Reversedphase high-performance liquid chromatographic determination of enoxacin and 4-oxo enoxacin in human plasma and prostatic tissue. Application to a pharmacokinetic study. J. Chromatogr.A $1998 ; 812: 369-75$.

16. Iglesias R., Villarroya F., Alemany M. Comparison of effects of different anticoagulants and sample handling procedures on rat insulin radioimmunoassay. Comp. Biochem. Physiol. A. $1985 ; 82: 863-6$.

17. Lagorce P., Perez Y., Ortiz J., Necciari J., Bressolle F. Assay method for the carboxylic acid metabolite of clopidogrel in human plasma by gas chromatography-mass spectrometry. J. Chromatogr. B Biomed. Sci. Appl.. $1998 ; 720: 107-17$.

18. Kummerle A., Krueger T., Dusmet M., Vallet C., Pan Y., Ris H.B., Decosterd L.A. A validated assay for measuring doxorubicin in biological fluids and tissues in an isolated lung perfusion model: matrix effect and heparin interference strongly influence doxorubicin measurements. J. Pharm. Biomed. Anal. 2003 ; 33 : 475-94.

19. Tangerman A: Highly sensitive gas chromatographic analysis of ethanol in whole blood, serum, urine, and fecal supernatants by the direct injection method. Clin. Chem.. $1997 ; 43: 1003-9$.

20. Robieux I., Aita P., Sorio R., Toffoli G., Boiocchi M. Determination of unbound etoposide concentration in ultrafiltered plasma by high-performance liquid chromatography with fluorimetric detection. J. Chromatogr. B Biomed. Appl. 1996 ; 686 : 35-41.

21. Combe B., Edno L., Lafforgue P., Bologna C., Bernard J.C., Acquaviva P., Sany J., Bressolle F. Total and free methotrexate pharmacokinetics, with and without piroxicam, in rheumatoid arthritis patients. Br. J. Rheumatol. $1995 ; 34: 421-8$.

22. Barré J., Chamouard J.M., Houin G., Tillement J.P. Equilibrium dialysis, ultrafiltration, and ultracentrifugation compared for determining the plasma-protein-binding characteristics of valproic acid. Clin. Chem. 1985 ; $31: 60-4$.

23. Oravcova J., Bohs B, Lindner W. Drug-protein binding sites. New trends in analytical and experimental methodology. J. Chromatogr. B Biomed. Appl..1996; 677 : 1-28. 
24. Blanchard I. Evaluation of the relative efficacy of various techniques for deproteinizing plasma samples prior to HPLC analysis. J. Chromatogr. B Biomed. Appl. 1981 ; $226: 455-60$.

25. Cherti N., Kinowski J.M., Lefrant J.Y., Bressolle F. High-performance liquid chromatographic determination of cefepime in human plasma, and urine and dialysate using a column-switching technique. J.Chromatogr. B Biomed. Appl. 2001 ; 754 : 377-86.

26. Kinowski J.M., Bressolle F. Fabre D., Goncalves F., Panis R., Galtier M. High-performance liquid chromatographic determination of ceftibuten and its metabolite in biological fluids. J. Pharm. Sci. 1994 ; 83 : 738-41.

27. Cociglio M., Hillaire-Buys D., Alric C. Determination of methotrexate and 7-hydroxymethotrexate by liquid chromatography for routine monitoring of plasma levels. J. Chromatogr, B Biomed. Appl. 1995 ; 674 : $101-10$.

28. Biddlecombe RA., Pleasance S. Automated protein precipitation by filtration in the 96-well format. $J$. Chromatogr. B Biomed. Appl. 1999 ; $734: 257-65$.

29. Cociglio M., Hillaire-Buys D., Peyriere H., Alric R. Performance analysis of a rapid HPLC determination with the solvent demixing extraction of HIV antiproteases and efavirenz in plasma. J. Chromatogr. Sci. $2003 ; 41: 80-6$.

30. Cociglio M., Alric R., Bouvier O. Performance analysis of a reversed-phase liquid chromatographic assay of lamotrigine in plasma using solvent-demixing extraction. J. Chromatogr. 1991; $572: 269-76$.

31. Cociglio M., Brandissou S., Alric R., Bressolle F. Highperformance liquid chromatographic determination of fluconazole in plasma. J. Chromatogr. B Biomed. Appl. $1996 ; 686: 11-7$.

32. Cociglio M., Hillaire-Buys D., Alric R. Prevalidation statistical design to assess analytical methods. Example of a quick liquid chromatographic assay of itraconazole in serum. J. Chromatogr. B Biomed. Sci. Appl. 1997 ; $698: 225-33$.

33. Srinivas N.D., Barhate R.S., Raghavarao K.S., Todd P. Acoustic demixing of aqueous two-phase systems. Appl. Microbiol. Biotechnol. $2000 ; 53$ : 650-4.

34. Srinivas N.D., Barhate R.S., Raghavarao K.S., Todd P. Acoustic field assisted enhanced demixing of aqueous two-phase systems. Biochim. Biophys. Acta. 2001 ; $1524: 38-44$.

35. Kelly M.T. Drug Analysis in Biological Fluids. In: Smyth MR (ed) Chemical Analysis in Complex Matrices. Chichester: Ellis Horwood. 1992.

36. Jemal M., Teitz D., Ouyang Z., Khan S. Comparison of plasma sample purification by manual liquid-liquid extraction, automated 96-well liquid-liquid extraction and automated 96-well solid-phase extraction for analysis by high-performance liquid chromatography with tandem mass spectrometry. J. Chromatogr. B Biomed. Sci. Appl. $1999 ; 732: 501-8$.

37. Zhang N., Hoffman K.L., Li W., Rossi D.T. Semi-automated 96-well liquid-liquid extraction for quantitation of drugs in biological fluids. J. Pharm. Biomed. Anal. $2000 ; 22 ; 131-8$
38. Ackermann B.L., Berna M.J., Murphy A.T. Recent advances in use of LC/MSMS for quantitative highthroughput bioanalytical support of drug discovery. Curr. Top. Med. Chem. $2002 ; 2: 53-66$.

39. Steinborner S., Henion J.. Liquid-liquid extraction in the 96-well plate format with SRM LC/MS quantitative determination of methotrexate and its major metabolite in human plasma. Anal. Chem.. $1999 ; 71: 2340-5$.

40.Peng S.X., Henson C., Strojnowski M.J., Golebiowski A., Klopfenstein S.R. Automated high-throughput liquidliquid extraction for initial purification of combinatorial libraries. Anal. Chem. 2000; $72: 261-6$.

41. Teitz D.S., Khan S., Powell M.L., Jemal M. An automated method of sample preparation of biofluids using pierceable caps to eliminate the uncapping of the sample tubes during sample transfer. J. Biochem. Biophys. Methods. $2000 ; 45$ : 193-204.

42. Zweigenbaum J., Heinig K., Steinborner S., Wachs T., Henion J. High-throughput bioanalytical LC/MS/MS determination of benzodiazepines in human urine: 1000 samples per 12 hours. Anal. Chem. 1999 ; 71 : 2294-300.

43. Jemal M. High-throughput quantitative bioanalysis by LC/MS/MS. Biomed. Chromatogr. 2000 ; 14 : 422-9.

44. Mahuzier C., Hamon M., Ferrier D., Prognon P. Chime analytique : méthodes de séparation. Abrégés de Pharmacie. Paris, Masson 1999.

45. Drummer O.H.. Chromatographic screening techniques in systematic toxicological analysis. J. Chromatogr. B Biomed. Sci. Appl. $1999 ; 733: 27-45$.

46. Rasanen I., Ojanpera I., Vuori E. Quantitative screening for benzodiazepines in blood by dual-column gas chromatography and comparison of the results with urine immunoassay. J. Anal. Toxicol. $2000 ; 24: 46-53$.

47. Pirnay S., Ricordel I., Libong D., Bouchonnet S. Sensitive method for the detection of 22 benzodiazepines by gas chromatography-ion trap tandem mass spectrometry. J. Chromatogr. A. $2002 ; 954: 235-45$.

48. Augey V., Cociglio M., Galtier M., Yearoo R., Pinsani V., Bressolle F. High-performance liquid chromatographic determination of cis-dichlorodiammineplatinum(II) in plasma ultrafiltrate. J. Pharm. Biomed. Anal. 1995; 13 : 1173-8.

49. Thurman E.M., Mills M.S. Solid-phase extraction: principles and practice. New York: John Wiley. 1998.

50. Walker V., Mills G. Solid-phase extraction in clinical biochemistry. Ann. Clin. Biochem. 2002 ; 39 : 464-77.

51. Franke J.P., de Zeeuw R. Solid-phase extraction procedures in systematic toxicological analysis. J. Chromatogr. B Biomed. Sci. Appl. $1998 ; 713: 51-9$.

52. Logan B.K., Stafford D.T. Liquid/solid extraction on diatomaceous earth for drug analysis in postmortem blood. J. Forensic. Sci. 1989 ; 34 : 553-64.

53. Lillsunde P., Korte T. Comprehensive drug screening in urine using solid-phase extraction and combined TLC and GC/MS identification. J. Anal. Toxicol. 1991; 15 : $71-81$. 
54. Pucci V., Bugamelli F., Mandrioli R., Bartoletti C., Rossi N., Ragg M. Liquid chromatographic analysis of the cis (Z) - and trans (E) -isomers of clopenthixol in human plasma using a novel solid phase extraction procedure. $J$. Chromatogr. B. Analyt. Technol. Biomed. Life Sci. $2003 ; 792: 313-21$.

55. Bergqvist Y., Funding L., Krysen B., Leek T., Yvell K., Improved validated assay for the determination of proguanil and its metabolites in plasma, whole blood, and urine using solid-phase extraction and high-performance liquid chromatography. Ther. Drug Monit. 1998 ; 20 : 325-30.

56. Casas M., Berrueta L., Gallo B., Vicente F. Solid-phase extraction of 1, 4-benzodiazepines from biologica fluids. J. Pharm. Biomed. Anal. $1993 ; 11: 277-84$.

57. Moors M., Massart D. Evaluation of solid-phase extraction of basic drugs from human milk. J. Pharm. Biomed. Anal. $1991 ; 9: 129-39$.

58. Bogusz M.J., Maier R.D., Kruger K.D., Kohls U. Determination of common drugs of abuse in body fluids using one isolation procedure and liquid chromatography-atmospheric-pressure chemical-ionization mass spectromery. J Anal Toxicol. $1998 ; 22$ : 549-58.

59. Ropero-Miller J.D., Lambing M.K., Winecker R.E. Simultaneous quantitation of opioids in blood by GC-EIMS analysis following deproteination, detautomerization of keto analytes, solid-phase extraction, and trimethylsilyl derivatization. J. Anal. Toxicol. 2002 ; 26 : 524-8.

60. Carson M.C.Ion-pair solid phase extraction. J. Chromatogr. A $2000 ; 885$ : 343-50.

61. Gustafson R., Moolchan E., Barnes A., Levine B., Huestis M. Validated method for the simultaneous determination of (9-tetrahydrocannabinol (THC), 11-hydroxyTHC and 11-nor-9-carboxy-THC in human plasma using solid phase extraction and gas chromatography-mass spectrometry with positive chemical ionization. $J$. Chromatogr. B. 2003; 798 : 145-54.

62. Chen X., Franke J.P., Ensing K., Wijsbeek J., de Zeeuw $\mathrm{R}$. Pitfalls and solutions in the development of a fully automated solid-phase extraction method for drug screening purposes in plasma and whole blood. J. Anal. Toxicol. $1993 ; 17: 421-6$.

63. Asselborn G., Yegles M., Wennig R. Suicide with reifentanil and midazolam: a case report. Acta. Clin. Belg. Suppl. $2002 ; 1: 54-7$.

64. de Zeeuw R., Wijsbeek J., Franke J.P. SPEC disc solidphase extraction for rapid broad-spectrum drug screening in urine. $2000 ; 2: 97-101$.

65. Stimpfl T., Jurenitsch J., Vycudilik W. General unknown screening in postmortem tissue and blood samples: a semi-automatic solid-phase extraction using polystyrene resins followed by liquid-liquid extraction. J. Anal. Toxicol. $2001 ; 25: 125-9$.

66 . Wells D. High throughput bioanalytical sample preparation - methods and automation strategies. Amsterdam: Elsevier Sci. BV. 2003.

67. Simpson H., Berthemy A., Buhrman D., Burton R. Newton J., Kealy M., Wells D., Wu D.. High throughput liquid chromatography/mass spectrometry bioanalysis using 96-well disk solid phase extraction plate for the sample preparation. Rapid Commun. Mass Spectrom. $1998 ; 12: 75-82$.
68. Boos K.S., Rudolphi A. The use of restricted-access media in HPLC, Part I - Classification and review. LCGC $1997 ; 15: 602-11$.

69.Boppana V:K., Miller-Stein C., Schaefer W.H. Direct plasma liquid chromatographic-tandem mass spectrometric analysis of granisetron and its 7-hydroxy metabolite utilizing internal surface reversed-phase guard columns and automated column switching devices. J. Chromatogr. B Biomed. Appl. 1996 ; 678 : 227-36.

70. Hsieh Y., Bryant M.S., Gruela G., Brisson J.M., Korfmacher W.A. Direct analysis of plasma samples for drug discovery compounds using mixed-function column liquid chromatography tandem mass spectrometry. Rapid. Commun. Mass Spectrom. 2000 ; 14 : 1384-90.

71. Ayrton J., Dear G.J., Leavens W.J., Mallett D.N., Plumb R.S. The use of turbulent flow chromatography/mass spectrometry for the rapid, direct analysis of a novel pharmaceutical compound in plasma. Rapid Commun. Mass Spectrom. $1997 ; 11: 1953-8$.

72. Vielhauer S., Rudolphi A., Boos K.S., Seidel D. Evaluation and routine application of the novel restrictedaccess precolumn packing material Alkyl-Diol Silica: coupled-column high-performance liquid chromatographic analysis of the photoreactive drug 8-methoxypsoralen in plasma. J. Chromatogr. B Biomed. Appl.. 1995 Apr $21 ; 666: 315-22$.

73. Sano T., Sato K., Kurihara R., Mizuno Y., Kojima T., Yamakawa Y., Yamada T., Ishii A., Katsumata Y. Sensitive determination of midazolam and identification of its two metabolites in human body fluids by columnswitching capillary high-performance liquid chromatography/fast atom bombardment-mass spectrometry. Leg. Med. $2001 ; 3: 149-56$.

74. Hayashida M., Hayakawa H., Wada K., Nihira M., Ohno $Y$. Sensitive determination of tetrodotoxin using columnswitching liquid chromatography-mass spectrometry with electrospray ionization in mouse serum. J. Anal. Toxicol. $2004 ; 28: 46-9$.

75. Ezquerro O., Pons B., Tena M.T. Evaluation of multiple solid-phase microextraction as a technique to remove the matrix effect in packaging analysis for determination of volatile organic compounds. J. Chromatogr. A. 2003 ; $1020: 189-97$.

76. de Toledo F.C., Yonamine M., de Moraes Moreau R.L., Silva O.A. Determination of cocaine, benzoylecgonine and cocaethylene in human hair by solid-phase microextraction and gas chromatography-mass spectrometry. J. Chromatogr. B Analyt. Technol. Biomed. Life Sci. $2003 ; 798: 361-5$.

77. Brown H., Kirkbride K.P., Pigou P.E., Walker G.S. New developments in SPME, Part 1: The use of vapor-phase deprotonation and on-fiber derivatization with alkylchloroformates in the analysis of preparations containing amphetamines. J. Forensic. Sci. $2003 ; 48: 1231-8$.

78. Ulrich S. Solid-phase microextraction in biomedical analysis. J. Chromatogr. A 2000 ; 902 : 167-94.

79. Degel F. Comparison of new solid-phase extraction methods for chromatographic identification of drugs in clinical toxicological analysis. Clin. Biochem. 1996 ; $29: 529-40$. 
80. Centini F, Masti A., Barni Comparini I..Quantitative and qualitative analysis of MDMA, MDEA, MA and amphetamine in urine by headspace/solid phase micro-extraction (SPME) and GC/MS. Forensic Sci. Int. 1996; 83 : 161-6.

81. Battu C., Marquet P., Fauconnet A.L., Lacassie E., Lachatre G. Screening procedure for 21 amphetaminerelated compounds in urine using solid-phase microextraction and gas chromatography-mass spectrometry. J. Chromatogr. Sci.. $1998 ; 36: 1-7$.

82. Nagasawa N., Yashiki M., Iwasaki Y., Hara K., Kojima T. Rapid analysis of amphetamines in blood using head space-solid phase microextraction and selected ion monitoring. Forensic Sci. Int. $1996 ; 78: 95-102$.

83. Benko A., Dona A., Kovacs A., Maravelias C., Mikone H.Z., Kerner A. Determination of amphetamine derivatives in urine with solid phase micro-extraction (SPME) Acta Pharm. Hung. $1998 ; 68: 269-75$.

84. Watanabe T., Namera A., Yashiki M., Iwasaki Y., Kojima T. Simple analysis of local anaesthetics in human blood using headspace solid-phase microextraction and gas chromatography-mass spectrometry-electron impact ionization selected ion monitoring. J. Chromatogr, B Biomed. Sci. Appl. 1998 ; 709 : 225-32.

85. Namera A., Watanabe T., Yashiki M., Iwasaki Y., Kojima T. Simple analysis of tetracyclic antidepressants in blood using headspace-solid-phase microextraction and GCMS. J. Anal. Toxicol. $1998 ; 22: 396-400$.

86. Lee X.P., Kumazawa T., Sato K., Suzuki O. Detection of tricyclic antidepressants in whole blood by headspace solid-phase microextraction and capillary gas chromatography. J. Chromatogr. Sci. $1997 ; 35$ : 302-8.

87. Ulrich S., Martens J. Solid-phase microextraction with capillary gas-liquid chromatography and nitrogen-phosphorus selective detection for the assay of antidepressant drugs in human plasma. J. Chromatogr. B Biomed. Sci. Appl. 1997 ; 696:217-34.

88. Kruggel S., Ulrich S. Solid-phase microextraction for the assay of levomepromazine in human plasma. Ther. Drug Monit. $2000 ; 22: 723-8$.

89. Hall B.J., Brodbelt J.S. Determination of barbiturates by solid-phase microextraction (SPME) and ion trap gas chromatography-mass spectrometry. J. Chromatogr. A. $1997 ; 777: 275-82$.
90. Guan F., Seno H., Ishii A., Watanabe K., Kumazawa T., Hattori H., Suzuki O. Solid-phase microextraction and GC-ECD of benzophenones for detection of benzodiazepines in urine. J. Anal. Toxicol. $1999 ; 23: 54-61$.

91. Frison G., Tedeschi L., Maietti S., Ferrara S.D.Determination of midazolam in human plasma by solid-phase microextraction and gas chromatography/mass spectrometry. Rapid Commun. Mass Spectrom. $2001 ; 15: 2497-501$.

92. Strano-Rossi S., Chiarotti M. Solid-phase microextraction for cannabinoids analysis in hair and its possible application to other drugs. J. Anal. Toxicol. $1999 ; 23: 7-10$.

93. Lachenmeier D.W., Kroener L., Musshoff F., Madea B. Application of tandem mass spectrometry combined with gas chromatography and headspace solid-phase dynamic extraction for the determination of drugs of abuse in hair samples. Rapid Commun. Mass Spectrom. 2003 ; 17 : 472-8.

94. Musshoff F., Junker H., Madea B. Simple determination of 22 organophosphorous pesticides in human blood using headspace solid-phase microextraction and gas chromatography with mass spectrometric detection. J. Chromatogr. Sci. $2002 ; 40: 29-34$.

95. Brewer W.E., Galipo R.C., Morgan S.L., Habben K.H. The confirmation of volatiles by solid-phase microextraction and GC-MS in the investigation of two traffic fatalities. J. Anal. Toxicol. $1997 ; 21: 286-90$.

96. Page B.D., Lacroix G. Application of solid-phase microextraction to the headspace gas chromatographic analysis of halogenated volatiles in selected foods. J. Chromatogr. $1993 ; 648: 199-211$.

97. De Martinis B.S., Martin C.C. Automated headspace solid-phase microextraction and capillary gas chromatography analysis of ethanol in postmortem specimens. Forensic Sci. Int.. $2002 ; 128: 115-9$.

98. Seno H., Ishii A., Watanabe K., Suzuki O., Kumazawa T. Extraction of chloroform and methylene chloride in human whole blood and urine by headspace solid phase microextraction (SPME). Med. Sci. Law. 1999 ; 39 : 332-6. 